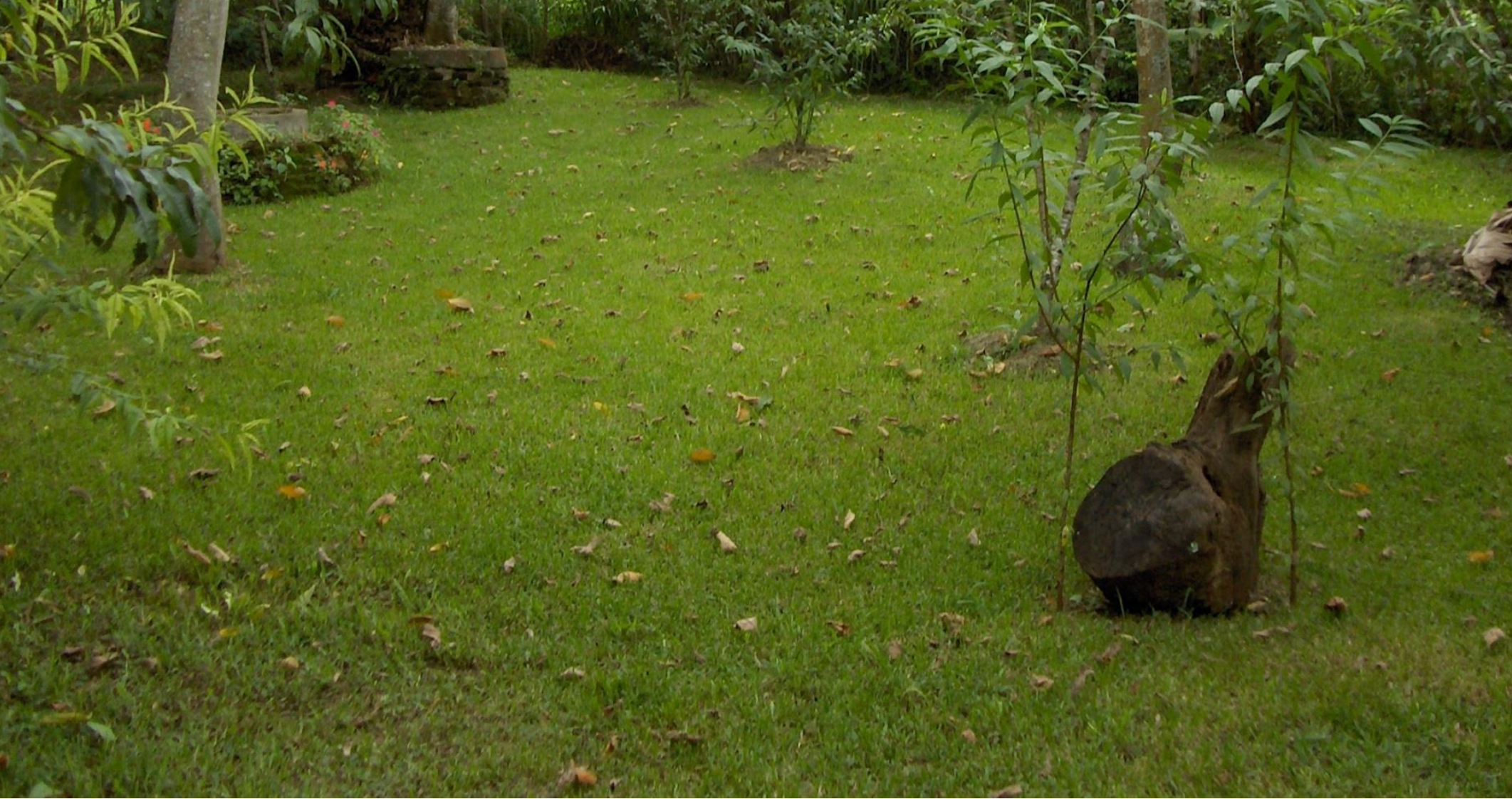

\title{
Modelo de promoción de la salud de CEnDHIU
}

José Guadalupe García González¹ - María José García Oramas²

RESUMEN: El Centro para el Desarrollo Humano e Integral de los Universitarios -CEnDHIU- es la entidad de la Universidad Veracruzana encargada de la "promoción de la salud y el fomento de entornos saludables" (Organización panamericana de la Salud, 1986). Mediante acciones de educación para la salud integral, servicios de salud mental, salud sexual, reproductiva, VIH, prevención de consumo de drogas lícitas e ilícitas, orientación psicopedagógica y atención personalizada a estudiantes viviendo con discapacidad. CEnDHIU contribuye a que la formación de los jóvenes no solo sea focalizada en lo técnico y profesional sino también con conciencia de la importancia de su propia salud, familia y comunidad para adquirir, durante su vida universitaria, estilos de vida saludable. Este trabajo muestra el modelo de promoción de salud implementado en esta entidad manteniendo actualizado el registro epidemiológico en estudiantes universitarios, así como sus principales resultados.

\footnotetext{
${ }^{1}$ Responsable Proyecto Programación y Diseño Institucional CEnDHIU, Universidad Veracruzana. Correo: guadagarcia@uv.mx.

${ }^{2}$ Coordinadora del Centro para el Desarrollo Humano e Integral de los Universitarios -CEnDHIU- Universidad veracruzana. Correo: jogarcia@uv.mx
} 
Palabras clave: promoción; salud; estudiantes; universitarios; centinelas.

\section{ABSTRACT:}

The Center for the Human and Integral Development of University Students -CEnDHIU-, is the entity of the Universidad Veracruzana responsible for the "promotion of health and the promotion of healthy environments" (Pan-American Health Organization, 1986). Through education actions for integral health, mental health services, sexual and reproductive health, HIV, prevention of licit and illicit drug use, psychopedagogical guidance and personalized attention to students living with disabilities, CEnDHIU contributes to the formation of young people not only be focused on the technical and professional but also with awareness of the importance of their own health, family and community to acquire healthy lifestyles during their university life. This work shows the model of health promotion implemented in this entity, keeping the epidemiological record in university students updated, as well as its main results.

Keywords: promotion; health; students; university; sentinels.

\section{Introducción}

A lo largo de los últimos veinte años, la Universidad Veracruzana ha trabajado en temas relativos al desarrollo académico con enfoque de calidad y desarrollo humano e integral, tarea realizada inicialmente por el Centro de Atención Integral para la salud del Estudiante Universitario -CENATI- desde 1992, cuya función principal era prevenir, rehabilitar y canalizar adecuadamente los problemas del estudiante y luego continuada, a partir del 2010, por Acuerdo Rectoral aprobado por Consejo Universitario, por el Centro para el Desarrollo Humano e Integral de los Universitarios -CEnDHIU-. El objetivo era fortalecer las tareas de CENATI, reorientarlas a fin de promover estilos de vida saludables y desarrollar un modelo de formación para los jóvenes y que no estuviese únicamente focalizado en el aspecto profesional, sino también en la conciencia de la importancia de su propia salud, la de su familia y de su comunidad, implementando para ello procesos innovadores de promoción de salud entre la comunidad universitaria.

De igual forma, "asegurar la equidad de oportunidades educativas de calidad para todos, independientemente de la situación socioeconómica, la raza, el género o, en su caso, la discapacidad de las y los alumnos" (Universidad Veracruzana, 2013). Apoyándoles de manera personalizada mediante el Programa Universitario de Educación Inclusiva.

De esta manera, el CEnDHIU tiene como objetivo promover una cultura de la salud entre las y los integrantes de la comunidad universitaria y para lograrlo, a partir del 2010 adopta la 
herramienta desarrollada por la Organización Mundial de la Salud para la vigilancia epidemiológica de problemas de salud denominada "centros centinelas" (Universidad Veracruzana, 2017), cuya propuesta básica es el desarrollo de competencias sociales e individuales a través de promover acciones para mejorar la salud y estilos de vida y brindar servicios accesibles en materia de salud en las propias facultades y centros de adscripción de los universitarios. En estos centros, se obtiene también información actualizada sobre factores de riesgo psicosociales para la construcción de indicadores y para monitorear las condiciones de salud de su red de cobertura relativos a estilos de vida, drogas lícitas e ilícitas, métodos anticonceptivos, salud mental autoestima, desarrollo humano, VIH sida.

\section{Objetivo}

Fomentar la promoción de la salud integral en la comunidad universitaria a partir de la detección oportuna de indicadores de salud.

\section{Materiales y métodos}

Se presentan los resultados obtenidos a partir de una investigación realizada con estudiantes universitarios aplicando un diseño estadístico previamente establecido para obtener el tamaño de muestra (n), donde inicialmente se aplicó un muestreo estratificado con asignación proporcional al tamaño, tomando como población objetivo (N) al número total de alumnos inscritos a partir del segundo semestre de la Universidad Veracruzana región Xalapa, en el periodo 2017-2018. (Tabla 1).

\section{$\mathrm{N}=22362$}

Tabla 1

Distribución de alumnos inscritos por estrato región Xalapa

\begin{tabular}{|l|c|}
\hline Estrato / Área académica & $\mathrm{Ni}$ \\
\hline Técnica & 4,777 \\
\hline Económico administrativa & 5,919 \\
\hline Humanidades & 5,106 \\
\hline Biológico-Agropecuaria & 1,355 \\
\hline Ciencias de la salud & 3,971 \\
\hline Artes & 1,234 \\
\hline Total & 22,362 \\
\hline
\end{tabular}


Con base en la siguiente expresión se calculó el tamaño de muestra para este estudio

$$
n=\frac{\sum \mathrm{Ni}^{2}(\text { piqi }) / \mathrm{W}}{N^{2} D+\sum N i p i q i}=378
$$

Donde:

$\mathrm{Ni}=$ número de alumnos inscritos en el i-esimo estrato (Área académica).

$\mathrm{Wi}=$ ponderación o peso en el estrato i.

$\mathrm{N}=$ número total de alumnos inscritos en el periodo.

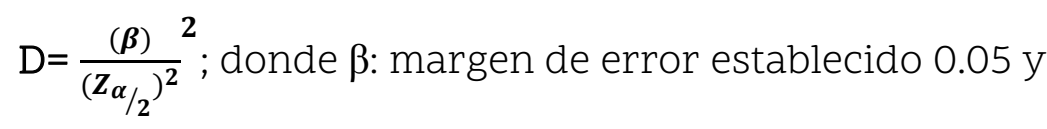

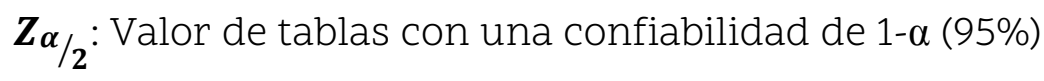

Posteriormente, en función al (n) obtenido se distribuyó la cantidad de estudiantes a entrevistar de manera proporcional por áreas Académicas y programas académicos, de acuerdo a la siguiente expresión: $n i=n(N i / N)$ o ni=n(W) (Tabla 2).

Tabla 2

Distribución de la muestra de acuerdo al área académica

\begin{tabular}{|l|c|c|}
\hline Estrato / Área académica & $\mathrm{Ni}$ & $\mathrm{ni}=\mathrm{n}(\mathrm{Ni} / \mathrm{N})$ o $\mathrm{ni}=\mathrm{n}(\mathrm{W})$ \\
\hline Técnica & 4,777 & 81 \\
\hline Económico administrativa & 5,919 & 100 \\
\hline Humanidades & 5,106 & 86 \\
\hline Biológico-Agropecuaria & 1,355 & 23 \\
\hline Ciencias de la salud & 3,971 & 67 \\
\hline Artes & 1,234 & 21 \\
\hline Total & 22,362 & 378 \\
\hline
\end{tabular}

Finalmente, fueron seleccionados aleatoriamente los estudiantes a los que se les aplicó el cuestionario siguiendo los criterios de inclusión al estudio para, posteriormente, proceder a su codificación, captura, validación de la base y análisis, cálculo de porcentajes, tablas de frecuencia y gráficos de pastel.

El cuestionario utilizado está compuesto por preguntas de opción múltiple y que fue diseñado expresamente para poder obtener información sobre factores de riesgo psicosocial tales como: 
estilo de vida, salud mental (específicamente depresión y ansiedad); consumo de drogas lícitas e ilícitas tales como: tabaco, alcohol, marihuana, cocaína, entre otras; salud sexual, tales como, inicio de vida sexual, uso de métodos anticonceptivos y condón. Algunos apartados hacen referencia a una parte del examen de salud que se aplica a los alumnos de nuevo ingreso, esto con la finalidad de tener un punto de comparación desde el ingreso hasta la conclusión de su vida universitaria.

La información se recolectó por personal responsable de los Centinelas, previamente capacitados, y finalmente se hizo la captura en la base de datos. A partir de ello se obtuvieron indicadores a fin de poder hacer inferencia en los factores de riesgo a evaluar y realizar acciones que favorezcan factores protectores.

\section{Resultados}

A continuación se presenta un concentrado de resultados de los factores que se consideran más significativos en razón de la trayectoria escolar de las y los alumnos, entre los cuales podemos destacar que 41\% de entrevistados presentan rasgos de depresión y 43\% de angustia. (Figura1 y figura 2).

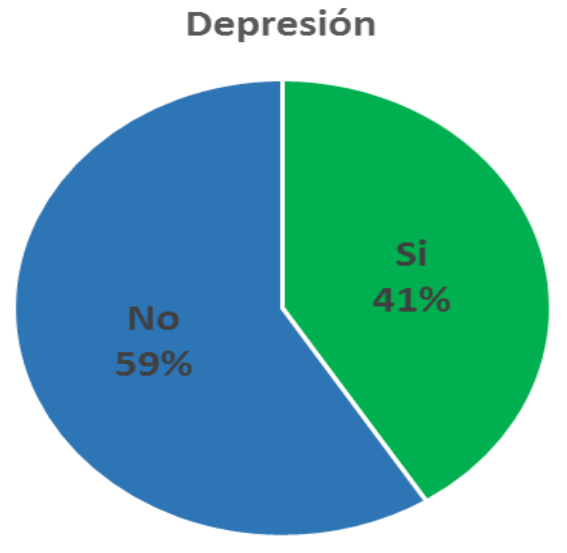

Figura 1. Alumnos con rasgos depresivos

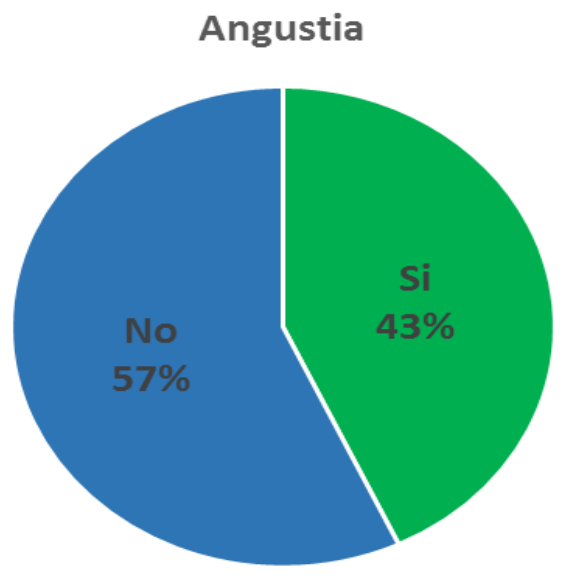

Figura 2. Alumnos con rasgos de angustia

Respecto a los estilos de vida, tenemos que el $47 \%$ presenta un estilo de vida muy adecuado y el $32 \%$ adecuado, pero existe un $17 \%$ de alumnos que pueden mejorarlo ya que están en rangos bajos y, más aún, el 1\% que está en la zona de peligro teniendo un estilo de vida inapropiado (figura 3). 


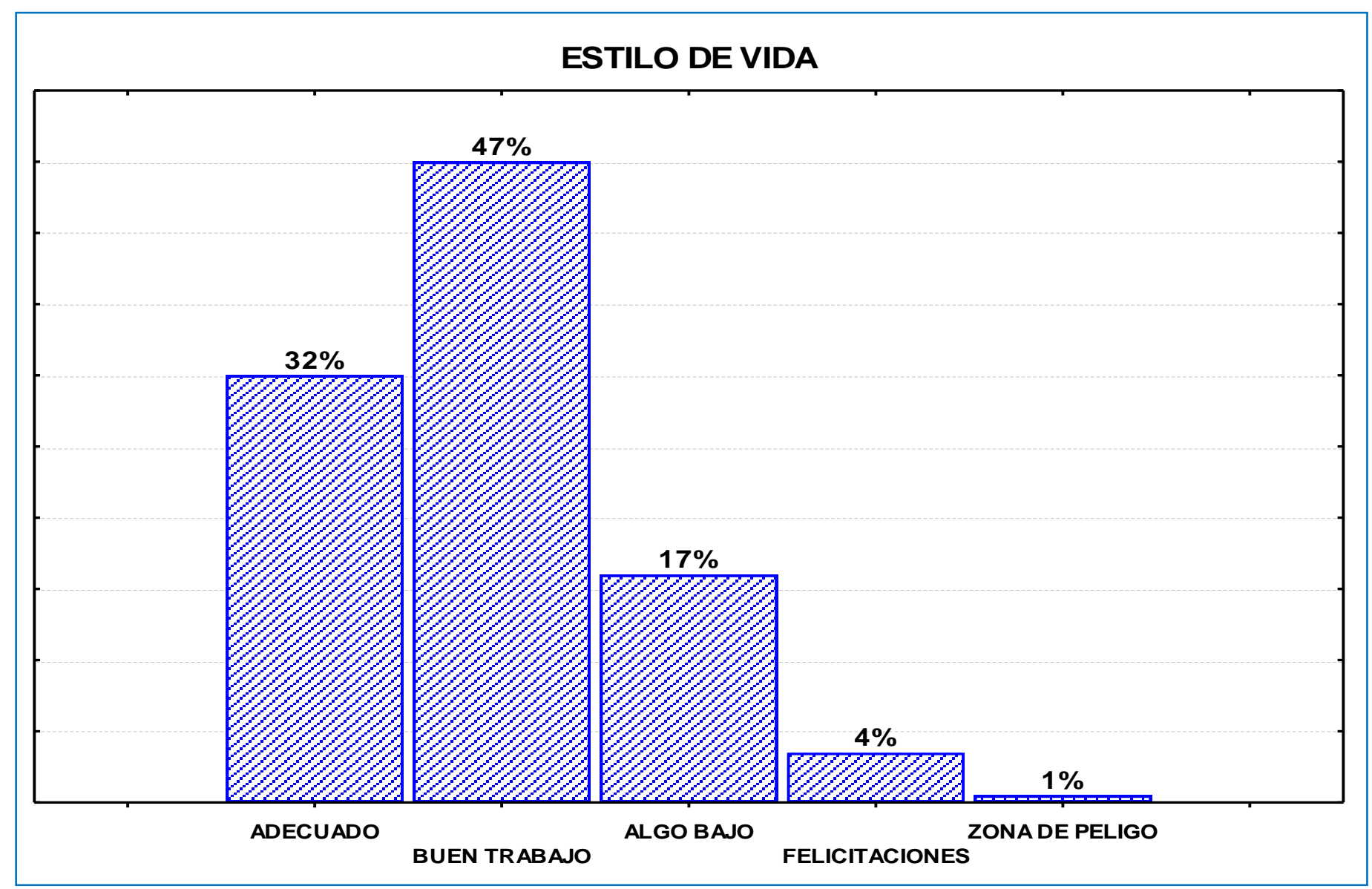

Figura 3. Distribución de los estilos de vida que presentaron los estudiantes.

En cuanto al consumo de drogas, observamos que el consumo de alcohol es el más común entre los estudiantes ya que el 81\% respondió consumirlo; en segundo lugar tenemos al consumo de tabaco con el $46 \%$ y en último lugar, aún en un porcentaje considerable, al 33\% que reporta consumo de drogas ilegales, destacando un 15\% que consumen la droga ilegal y lo hacen recurrentemente en un periodo mínimo a un mes. (Tabla 3).

Tabla 3

Proporción de alumnos que consumen drogas

\begin{tabular}{|l|c|}
\hline Consumo de drogas & Porcentaje \\
\hline Fuma & $46 \%$ \\
\hline Ha fumado más de 5 Cajetillas en su vida & $23 \%$ \\
\hline Consumo de alcohol & $81 \%$ \\
\hline Drogas ilegales & $33 \%$ \\
\hline Prevalencia lápsica de consumo de drogas ilegales últimos 30 días & $15 \%$ \\
\hline
\end{tabular}


En lo que respecta a la vida sexual, 83\% de estudiantes reportan iniciarla a los 18 años, período en que ya son integrantes de la comunidad universitaria. Sin embargo, siguen mostrando conductas de riesgo ya que el 62\% no usó condón en su última relación sexual y con ello están expuestos a contraer una ITS o a un embarazo no planeado (Tabla 4).

\section{Tabla 4}

Proporción de alumnos sexualmente activos y uso de condón

\begin{tabular}{|l|c|}
\hline Sexualidad & Porcentaje \\
\hline Vida sexual activa & $83 \%$ \\
\hline Años promedio Inicio de vida sexual & 18 \\
\hline Usó condón en su última relación sexual & $62 \%$ \\
\hline
\end{tabular}

\section{Conclusiones}

A partir de estos resultados, se busca reorientar los proyectos y las estrategias de difusión y promoción de la salud integral implementadas en el CEnDHIU a fin de fortalecer factores protectores, a través de los denominados centros centinela y módulos informativos, mediante cursos, talleres, experiencias educativas, capacitaciones, fiestas de la salud; aplicación voluntaria de pruebas gratuitas de VIH, asesoría y dotación de métodos anticonceptivos gratuitos; consejería psicológica y psicopedagógica oportuna, prevención de consumo de drogas legales e ilegales, campañas de salud y el programa de radio "Llégale aquí estamos".

Se considera que CEnDHIU ha creado un marco común para la promoción, la prevención y atención oportuna en materia de promoción de la salud ${ }^{3}$ integral para las y los universitarios destacando conductas de riesgo y fortaleciendo factores protectores, desde un modelo de participación de la comunidad universitaria implicando activamente a todas y todos sus actores.

\section{Referencias:}

Organización Panamericana de la Salud. (1986). Carta de Ottawa. Recuperado de https://www.paho.org/hq/dmdocuments/2013/Carta-de-ottawa-para-la-apromocion-de-la-salud1986-SP.pdf.

Universidad Veracruzana. (2013). Programa de Trabajo Estratégico 2013-2017. Recuperado de https://www.uv.mx/programa-trabajo/Programa-de-Trabajo-Estrategico-version-parapantalla.pdf 
Universidad Veracruzana. (2017). Programa Centro Centinela Cendhiu. Recuperado de https://www.uv.mx/cendhiu/files/2016/10/PROGRAMA-CENTRO-CENTINELA-2018.pdf 